\title{
Analytical Framework for Market-oriented DSR Flexibility Integration and Management
}

\author{
Shi You, Junjie Hu, Kai Heussen, Chunyu Zhang \\ Department of Electrical Engineering, Technical University of Denmark, Lyngby, Denmark \\ Email: sy@elektro.dtu.dk, \{junhu, kh, chzh\}@elektro.dtu.dk
}

Received March, 2013

\begin{abstract}
Integration and management of the flexibility of Demand Side Resources (DSR) in today’s energy systems plays a significant role in building up a sustainable society. However, the challenges of understanding, predicating and handling the uncertainties associated this subject to a great extent hamper its development. In this paper, an analytical framework based on a multi-portfolio setup in presence of a deregulated power market is proposed to address such challenges by adopting the thinking in modern portfolio theory (MPT). A Numerical example that targets on analyzing the risk and return for various flexibility pricing strategies are presented to illustrate some features of the framework.
\end{abstract}

Keywords: Analytical Framework; Distributed Energy Resources; Flexiblity; Deregulated Power Market; Morden Portfolio Theory; Risk and Return

\section{Introduction}

Demand side resource (DSR) refers to the geographically distributed modular power generation, consumption and energy storage systems which are located on the demand side and have the capability of altering their generation/consumption pattern. The capability of DSR, also referred as flexibility, has been considered as perpetual resources with significant value of both technical and economic prospects for reducing energy consumption, improving energy efficiency, facilitating the integration of stochastic renewable, deferring the expansion of power networks and securing the power system operation via ancillary services' provision, etc. [1,2]

Conventional demand response (DR) programs are normally organized by utilities, and can be split in two categories. The first group requires a fast and reliable response from DR programs, so that the DSRs are remotely controlled by the utilities in a master-slave manner under bilateral agreements. These programs primarily target on medium to large size commercial and industrial DSRs and they are typically limited to interruptible DR services. A second group of DR programs is aimed at modifying the consumption of a large number of smallscale DSRs by means of economic incentives. These so called dynamic tariff programs include e.g. hourly real time pricing (RTP) and time-of-use tariffs (TOU) but

\footnotetext{
${ }^{1}$ http://www.ipower-net.dk/

${ }^{2}$ FlexPower homepage hosted by Ea Energianalyse

${ }^{3}$ http://www.eu-ecogrid.net/
}

also capacity pricing are used to shift consumption patterns and achieve better system economics with 'dynamics' in the scale of hours to years [3].

Along with the anticipated increase in penetration of DSR in the distribution systems, both the utilities and the emerging non-utility entities, e.g. Virtual Power Plants (VPPs), DSR aggregators, intent on exploiting the added value of DSR flexibility [4,5]. By coordinating a fast response from these low cost small-scale DSRs by means of various advanced control strategies, the aggregated DSR flexibility could be treated the same as other demand/generation resources in the context of a deregulated electricity market. However, integrating the DSR flexibility into the power system operation through the present deregulated market setup is challenged by a number of uncertain factors, such as

- the wide range of DSR technologies with varying flexibility options;

- the diversity of aggression-oriented control strategies with few comparisons among them due to the flexibility of control architectures and the vaguely defined evaluation criteria , etc.;

- the multiple-choice of market products (e.g. ancillary services) with different risk-return spectrum and requirements.

Using Denmark as a test field, several ongoing projects, e.g. iPower $^{1}$, Flexpower ${ }^{2}$ and EcogridEU ${ }^{3}$, are working on understanding, predicating and handling the uncertainties associated with market-oriented DSR flexibility integration and management. In these projects, 
solutions are investigated from technical, economic and social perspectives, where the interests of different stakeholders, i.e. Transmission System Operators (TSOs), Distribution System Operators (DSOs), Balance Responsible Parties (BRPs, here to be considered mostly equivalent to 'aggregators') and DSR owners, are balanced in the context of the Nordic power market.

In this paper, an analytical framework based on a multi-portfolio setup is proposed to address such challenges by adopting the thinking in modern portfolio theory (MPT). Such a multi-portfolio framework is to analyze the risk and return for different portfolio mixes from both economic and technical perspectives. This paper is organized as follows. First, a brief interpretation of this analytical framework is provided in Section II. Section III presents the relevant techniques for constructing the multi-portfolio setup. A numerical example that targets on analyzing the risk and return for different flexibility pricing strategies is presented in Section IV to illustrate some features of the framework, while Section V discusses the chances and challenges of further development of this framework and concludes.

\section{A Multi-portfolio Analitical Framework for DSR Flexibility Management and Integration}

A multi-portfolio based analytical framework for DSR flexibility management and integration is illustrated in Figure 1. Different stakeholders mentioned earlier can establish their interest-oriented portfolios by the use of experimental or model-based setups; meanwhile the corresponding risk-return performance indicators can be identified or even created to satisfy the stakeholder's individual interest. Different techniques for portfolio analysis, e.g. MPT, can be applied to performing the relevant analysis, and the analytical results will be fed back to the stakeholders who need to decide on if further actions, e.g. reconfiguring portfolio setups, alternating the performance indicators, are required.

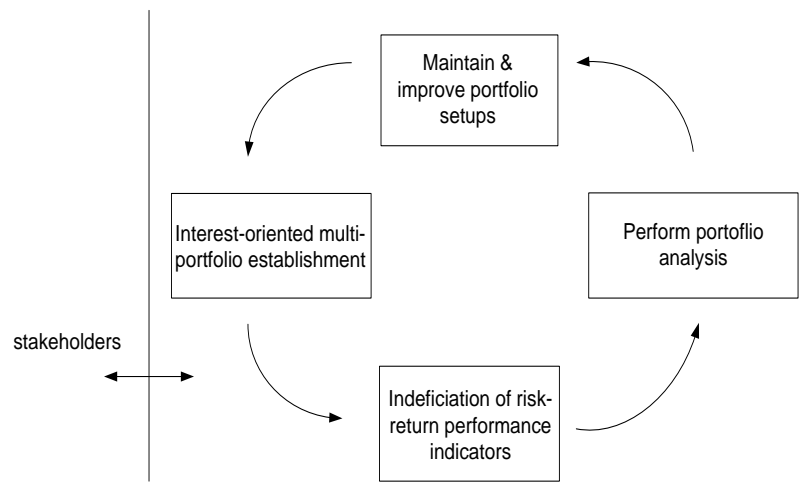

Figure 1. Multi-portofolio based analytical framework for DSR flexibility management and integration.
In general, portfolio analysis is applied to financial portfolios which are combinations of various financial products such as bonds, equities, indexes, funds, and securities. It involves quantifying the expected financial returns for different portfolios and the associated risk which is often expressed as volatility of returns. For its application to DSR flexibility management and integration, new measures of risk and return can be defined. For instance, when a BRP with a given DSR portfolio would like to provide frequency control ancillary services and are in doubt with the optimal combination of its control strategies, in addition to using the economic metrics, the risk associated with control performance could also be taken into account. For another example, if the local energy suppliers would like to investigate a portfolio with different electricity pricing methods, e.g. fixed-price (FP), time of use (TOU), real time price (RTP), the risk and return on customer satisfaction might also be considered.

In Figure 2, an illustrative example with a threeportfolio analytical setup is presented. In this setup, the uncertainties of market-oriented DSR flexibility management and integration are treated in three closely related portfolios, wherein DSR flexibility, control strategy and market-based flexibility service are grouped separately. Each portfolio can be seen as a combination of weighted assets those belong to the corresponding portfolio category, so that the return of a portfolio is the weighted combination of the assets' returns. By changing the percentage of one/several asset in a portfolio, the peculiar risk associated with these assets and returns associated with them can be characterized and analyzed in different ways. In rest of the paper, analysis and discussion related to using this analytical framework to facilitate market-based DSR flexibility management and integration will be referred to this three-portfolio setup.

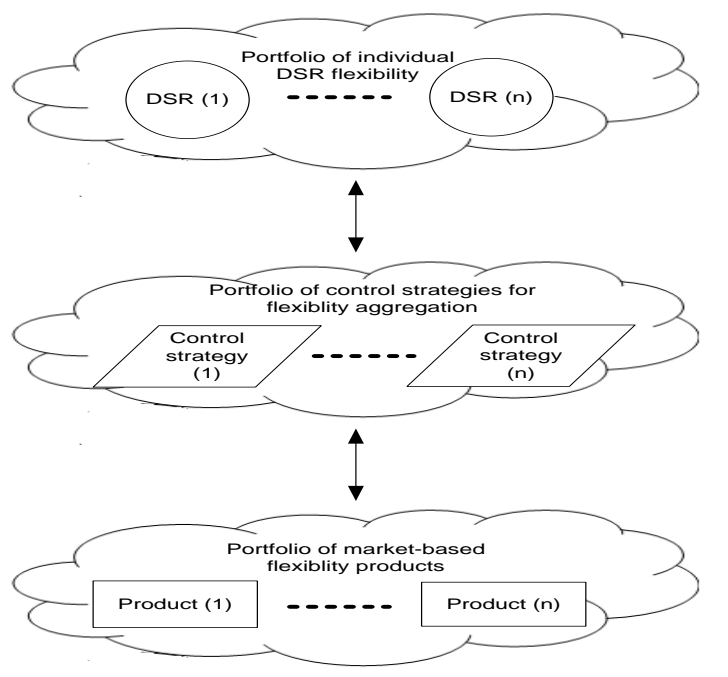

Figure 2. A three-portofolio establishment for DSR flexibil- 
ity management and integration.

It is worthwhile to note that, this three-portfolio-based analytical setup only illustrates a conceptual example of the generic analytical framework. Portfolios of ICT solutions, etc., can also be included. The process of investigating the variability of portfolio setups and the associated risk-return is analogous to the process of modeling a constrained multi-objective optimization problem and finding its optimal solutions, wherein exogenous and endogenous variables as well as different objectives have to be selected, characterized and regulated with care.

\section{Establishing the Multi-portfolio Setup}

\subsection{Portfolio of DSR Flexiblity}

The quantitative flexibility of a DSR, i.e. when and how much can its generation/consumption pattern be alternated, is determined by its local control system. In many cases, this decision making process needs to consider exogenous inputs (e.g. knowledge of global and local environment) and endogenous inputs (e.g. knowledge of the DSR plant dynamics and physical constraints), as well the local objectives (e.g. utility maximization or cost minimization). The generic formulation of DSR flexibility is hereby expressed, as in (1).

$$
\begin{gathered}
x(q, t)=\arg \max u\left(x^{*}\right) \\
\text { s.t. } \Phi(x)
\end{gathered}
$$

where $x(q, t)$ stands for the flexibility of a DSR device describing its output of active power $q$ at time $t ; u(x)$ stands for the DSR owner's utility; $\Phi(x)$ stands for all necessary constraints.

This generic expression is more applicable to modeling the flexibility of an individual DSR; meanwhile it can be used to derive the optimal generation/consumption schedule for a certain period. When aggregated flexibility over a certain amount of DSR is to be modeled, statistical techniques, e.g. Monte Carlo method, can be applied based on direct observations of individual DSR. It is important for the modelers to recognize the cross-time feature of DSR flexibility, particularly for the DSRs with deferrable characteristics, e.g. electric vehicles, thermal storages. In other words, the DSR flexibility is not always time invariant.

\subsection{Portfolio of Control-strategies}

A control strategy is a set of specific measures identified and implemented to achieve the control objective. For an aggregation-oriented control strategy, it is consisted of a set of important features such as control patterns (e.g. open-loop vs. close-loop), control structures (e.g. centralized vs. decentralized) and control algorithms (e.g. model-predictive control vs. standard PID control), which need to be carefully designed and integrated in a control system architecture.

One conceptual way for classifying different aggregation-oriented control strategies is given in [6], wherein the two categories, namely "direct control" and "indirect control” are thoroughly introduced. The former alludes to a conventional control approach which requires DSR state information to compute reference trajectories for the DSR consumption to follow. The latter approach is often associated with broadcasting of incentive signals (prices) with an update frequency of e.g. 5 minutes to the DSRs. Compared to the hourly dynamic tariffs, this update frequency is fast, and falls into the time range of generator ramps, for example.

This way of classification is in line with the commonly-understood way for classifying and modeling the controllability of DSR, i.e. price-responsive and controllable (price signal free) as depicted in Table 1; meanwhile, the systematical structuring for "directness" and "indirectness" could particularly support the active demand side management by combining the control engineering domain with the value-oriented deliberation and addressing the integration and valuation of mixed portfolios of direct and indirect control as well as the further analysis of co-existence of such control-solutions with respect to overall control architecture of the power system.

\subsection{Portfolio of Market Products}

The needs which can exploit the DSR flexibility for achieving the objectives of different stakeholders are many and they vary in requirements on volume, location, time and reliability, etc. In a liberalized market, ideally these system needs are transformed into different market products which will influence the way of flexibility integration and management. For the needs relating to achieving the reliable, secure and efficient operation of electric power systems, although the market-based setups vary across countries [7], many of them have already been reflected in existing market products such as

Table 1. A brief overview of eamples \& techniques for con-

\begin{tabular}{|c|c|c|c|}
\hline & \multirow[b]{2}{*}{$\begin{array}{l}\text { Price-signal } \\
\text { free }\end{array}$} & \multicolumn{2}{|c|}{ Price-signal enabled } \\
\hline & & $\begin{array}{c}\text { Double-sided } \\
\text { auction } \\
\text { (coordination) }\end{array}$ & $\begin{array}{c}\text { Single-sided } \\
\text { auction } \\
\text { (incentive) }\end{array}$ \\
\hline Deterministic & $\begin{array}{l}\text { Conventional } \\
\text { control, e.g. } \\
\text { load shedding }\end{array}$ & $\begin{array}{l}\text { Market clearing } \\
\text { on the basis of } \\
\text { bids and offers }\end{array}$ & $\begin{array}{c}\text { Pricing } \\
\text { schemes, e.g. } \\
\text { TOU, RTP }\end{array}$ \\
\hline Probabilistic & $\begin{array}{l}\text { Aggregated } \\
\text { TCLs }{ }^{a} \text { controlled by } \\
\text { freq./temp. set-points }\end{array}$ & $\begin{array}{c}\text { Game theory } \\
\text { enabled stochastic } \\
\text { modeling }\end{array}$ & $\begin{array}{l}\text { Homeostatic } \\
\text { utility control }\end{array}$ \\
\hline
\end{tabular}
trol strategies modeling. 
- $\quad$ Capacity products for DSOs and TSOs to ensure the resource adequacy.

- Emergency products for DSOs and TSOs to handle emergency situations.

- Ancillary service products for TSOs including frequency regulation, voltage control, short circuit capacity, manual reserve, black-start, etc.

- Service products for DSOs, e.g. peak shaving, voltage control, congestion management, etc.

- Energy balancing products for BRPs to maintain their balancing responsibilities.

Techniques for modeling and analyzing the economic performance for the electrical power market and its associated products based on observed market behavior have been extensively developed and implemented [8].However, in addition to investigating the economic performance of different products, it is also important to analyze the technical requirements for each market product in order to find appropriate portfolio mappings among DSR flexibility, control strategies and the market products.

\section{Numerical Example}

In this section, a numerical example of peak load reduction is presented to illustrate some features of the proposed analytical framework. Data of residential loads and wholesale electricity prices used in this example are provided by the Danish DSO and the Danish TSO respectively, corresponding to the observations in 2011.

Assuming in one $0.4 \mathrm{kV}$ radial distribution feeder, 42 Danish single-family households constitute the electrical load of this feeder. Local DSO would like to investigate, comparing with the current FP scheme, how much can other different pricing schemes affect the maximum loading over the feeder system and thereby conducts the following study.

The 42 households load profiles are modeled based on their previous performance, wherein an annual consumption of $4000 \mathrm{kWh}$ per household was observed under FP. Assumption about the flexibility of each household is made, i.e. the price elasticity is set as -1 while the maximum flexibility of each household at each hour can be $\pm 30 \%$ of the corresponding hourly load under FP.

Regarding the variety of pricing schemes, as in Figure 3 , the hourly wholesale electricity spot is assigned as RTP; FP is assumed to be the annual average of RTP; while TOU is comprised of three periods: peak (7-9 and 17-19), shoulder (4-6, 10-16 and 20-21) and off-peak (1-3 and 22-24). For calculating the TOU, the electricity price of each period is derived by averaging the spot prices over all those hours t belong to that period category over the year.

After simulating different pricing schemes, the re- sulted daily peak load over the year under different context is derived as in Figure 4. Compared with the existing FP, TOU to some extent lowers the daily peak while RTP introduces both lower and higher peak values due to the demand side elasticity.

To further analyze the three pricing schemes constituted control strategy portfolio, an efficient frontier (i.e. the blue curved line) is plotted as in Figure 5. The concept of efficient frontiers was introduced in 1952 by Nobel Prize winner Harry Markowitz as part of the Capital Asset Pricing Model (CAPM) for portfolio theory[9].A key finding of the concept was the benefit of diversification as depicted in Figure 5 by the random portfolio asset combinations given in red dots, while the principle shows that combing several stocks into a portfolio can decrease the overall risk below that of any individual stock while still attaining a comparable return. The efficient frontier therefore gives the lowest level of risk needed to achieve a given expected rate of return or the

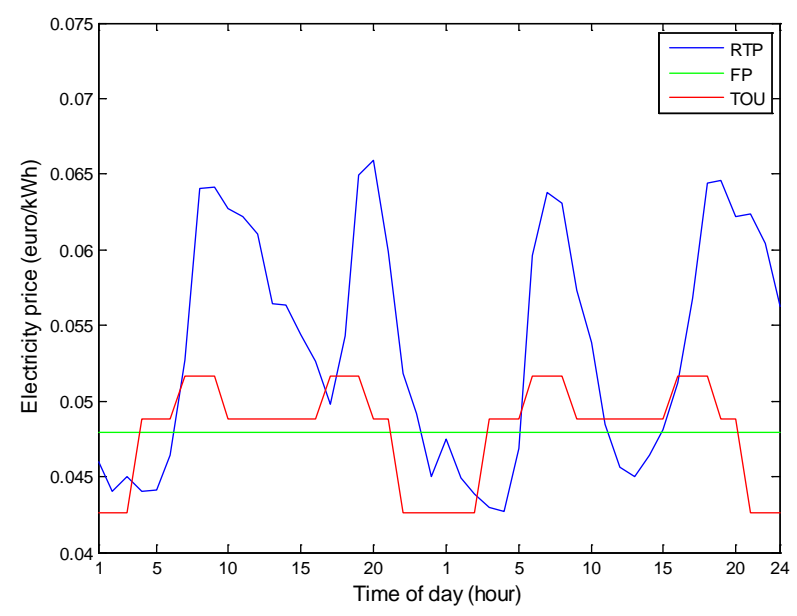

Figure 3. Different pricing shcemes in two consecutive days.

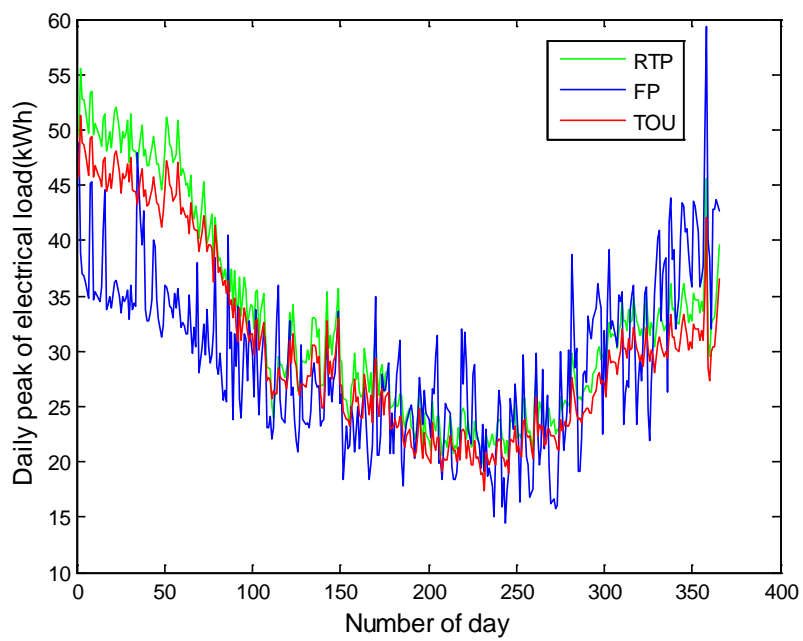

Figure 4. Daily peak load over the year under different pricing schemes. 


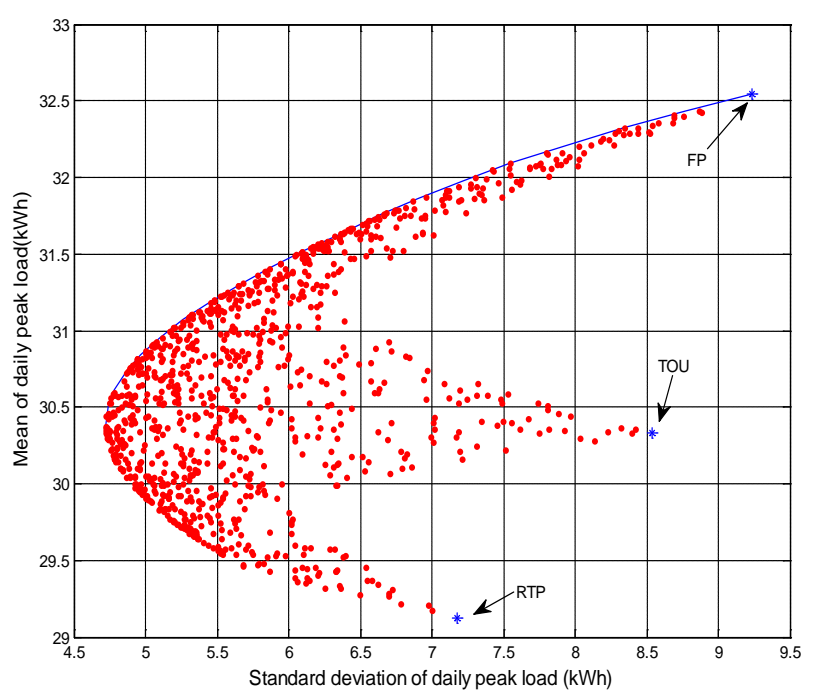

Figure 5. Mean-variance efficient frontier and random combinations for the three different pricing schemes.

best return that can be expected for a given level of risk. In the presented example, it can be found that FP, TOU and RTP exhibit a decreasing order with respect to both the mean values of daily peaks and the associated standard deviations. The optimal portfolio with minimum risk (i.e. measured by standard deviation) is found with a combination of $25.9 \%$ FP, $43.7 \%$ RTP and $30.4 \%$ TOU, which results in a mean of daily peak at $30.38 \mathrm{kWh}$ with standard deviation of $4.72 \mathrm{kWh}$.

\section{Discussion and Conclusions}

A successful deployment of the emerging technologies in smart grid and smart market require a deep understanding of the associated risk and return for different stakeholders. In the complex electrical energy system, the inseparable relationships across various domains and different stakeholders make the problem even more challenging. The paper proposes a multi-portfolio-based analytical framework for market-oriented DSR integration and management. This framework aims at clarifying the tradeoffs in satisfying different stakeholders' objectives and acceptable risk, or variability of tradeoffs.

By itself, the proposed framework can be easily understood and might result in clear suggestions to various stakeholders given their concerns, priorities and re- sources. However, in reality, the stakeholders generally have to face many choices and handle the worst cases, which would require them to put more effort on structured thinking, detailed modeling and careful analysis when to use the framework.

\section{Acknowledgements}

The authors would like to thank the Danish iPower platform for its financial support on this study.

\section{REFERENCES}

[1] G. Strbac, "Demand Side Management: Benefits and Challenges,” Energy Policy, Vol. 36, No. 12, 2008, pp. 4419-4426. doi:10.1016/j.enpol.2008.09.030

[2] P. Palensky, "Demand Side Management: Demand Response, Intelligent Energy Systems, and Smart Loads," IEEE Transactions on Industrial Informatics, Vol. 7, No. 3, 2011, pp. 381-388. doi:10.1109/TII.2011.2158841

[3] S. Borenstein and M. Jaske, "Dynamic Pricing, Advanced Metering, and Demand Response in Electricity Markets,” Project Report, Center for the Study of Energy Market, University of California, 2002.

[4] S. You, C. Træholt and B. Poulsen, "A Market-based Virtual Power Plant," in Proceedings of International Conference on Clean Electrical Power, 2009, pp. 460-465.

[5] S. You, C. Træholt and B. Poulsen, "Gemeroc Virtual Power Plants: Management of Distributed Energy Resources under Liberlized Electricity Market," in Proceedings of the 8th IET International Conferences on Advances in Power System Control, Operation and Mnagement, 2009, pp. 1-6.

[6] K. Hesussen, S. You and B. Biegel, "Indirect Control for Demand Side Management - A Conceptual Introduction,” in Proceedings of 2012 IEE PES Innovative Smart Grid Technologies.

[7] P. Nyeng, "System Integration of Distributed Energy Resouces,” PhD Thesis, Technical University of Denmark, 2010

[8] M. Ventosa, A. Baillo, A. Ramos and M. Rivier, "Electricity Market Modeling Trends,” Energy Policy, Vol. 33, No. 7, 2005, pp. 897-913.

$$
\text { doi:10.1016/j.enpol.2003.10.013 }
$$

[9] E. J. Elton, M. J. Gruber, S. J. Brown and W. N. Goetzmann, Morden Portfolio Theory and Investment Analysis, John Wiley \& Sons, Inc., 7th edition, 2007. 